\title{
Jungquartäre Flussterrassen am mittleren Lech zwischen Kinsau und Klosterlechfeld - Erste Ergebnisse
}

\author{
Benjamin Geßlein, Gerhard Schellmann
}

Abstract:

\begin{abstract}
GPS based field mappings of the fluvial terraces of the river Lech between Kinsau and Klosterlechfeld demonstrate a stratigraphic differentiation of the younger Quaternary valley bottom by a terrace flight of at least 17 Lech terraces. The oldest terrace of probably Early to Middle Würmian age is the Übergangsterrasse (transitional terrace), which is in contrast to the younger Würmian and Holocene terraces covered by a thin layer of sandy loess sediments. The valley is dominated by six Würmian Pleni- and Lateglacial terraces which engage about $65 \%$ of the valley plain. While the oldest Lower Terrace, the Hauptniederterrasse including its subfields, is directly connected to the terminal moraines of the Würmian Last Glacial Maximum (LGM) both younger Late Pleniglacial lower terraces (Stufe von Altenstadt, Stufe von Schongau-Peiting) have no direct connection to a terminal moraine. The Würmian Lateglacial terraces, the Stufe von Unterigling, the Zwischenstufe and the Stufe von Friedheim, are the youngest lower terraces and in a morphological sense they mark the transition from the Pleniglacial to the Holocene terraces. The Holocene terraces incorporate up to ten differently elevated channel deposits. While the oldest Holocene terrace was formed during the early Preboreal the three youngest ones were built from Roman to Modern age.
\end{abstract}

\section{[Late Quaternary river terraces at the middle reaches of the river Lech - first results]}

Kurzfassung:
Im Rahmen einer geomorphologisch/quartärgeologischen Neuaufnahme der Lechterrassen zwischen Kinsau und Klosterlech- feld konnte die bisher bekannte jungpleistozäne Terrassentreppe neu aufgenommen und teilweise revidiert werden. Nach ak- tuellem Stand umfasst diese insgesamt 17 morphologisch voneinander abgesetzte Terrassenkörper. Die chronostratigraphische Einordnung der Lechterrassen stützt sich vor allem auf morphostratigraphische Befunde sowie einzelne absolute Altersdatie- rungen $\left({ }^{14} \mathrm{C}\right)$ an Schneckenschalen und Holzfragmenten aus deren Flussbettsedimenten bzw. an Flusssanden (OSL).
Die älteste bisher bekannte früh- bis mittelwürmzeitliche Lechterrasse wird als „Übergangsterrasse“ bezeichnet. Sie besitzt, im Gegensatz zu den jüngeren Lechterrassen, eine etwa einen Meter mächtige Deckschicht aus sandstreifigem Löss. Sie ist älter als die hochglaziale, über Schmelzwasserbahnen mit den würmhochglazialen Jungendmoränenständen im Raum Hohenfurch verknüpfte Hauptniederterrasse (HNT).
Die Hauptniederterrasse und ihre Teilfelder nehmen flächenmäßig bis zu 65\% des Talgrundes südlich von Landsberg ein und sind mit der würmhochglazialen äußeren Jungendmoräne verbunden. Dagegen ist eine direkte morphologische Verbindung der späthochglazialen Niederterrassen (Stufe von Altenstadt, Stufe von Schongau-Peiting) mit würmzeitlichen Abschmelzständen nicht mehr erhalten. Neben der Hauptniederterrasse und ihren Teilfeldern sowie den beiden späthochglazialen Niederterras- sen (Stufen von Altenstadt, Stufe von Schongau-Peiting) existieren drei weitere spätglaziale Niederterrassen, die Stufe von Unterigling, die Zwischenstufe und die sogenannte Stufe von Friedheim. Nach der ${ }^{14} \mathrm{C}-$ Datierung einer Schneckenschale dürfte die jüngste der drei Stufen, die Stufe von Friedheim, eine jüngerdryaszeitliche Bildung sein, die noch vor etwa 10120 a BP in Ausbildung begriffen war. Die holozänen Terrassen bilden ebenfalls eine Terrassentreppe mit bis zu zehn einzelnen Stufen, die im Zeitraum vom frühen Präboreal bis in die jüngere Neuzeit entstanden sind.

Keywords: $\quad$ Lechtal, Terraces, Late Pleistocene, Holocene, Anabranching River, Braided River

Addresses of authors: B. Gesslein, Universität Bamberg, Institut für Geographie, Lehrstuhl II für Physische Geographie, Am Kranen 1, 96045 Bamberg. E-Mail: benjamin.gesslein@uni-bamberg.de; G. Schellmann, Universität Bamberg, Institut für Geographie, Lehrstuhl II für Physische Geographie, Am Kranen 1, 96045 Bamberg. E-Mail: gerhard.schellmann@uni-bamberg.de

\section{Einführung in das Untersuchungsgebiet}

Das Untersuchungsgebiet umfasst das mittlere Lechtal nördlich des Engtaldurchbruchs durch die würmhochglazialen Jungendmoränen nahe Kinsau und reicht etwa $35 \mathrm{~km}$ talabwärts bis nach Klosterlechfeld südlich von Augsburg (Abb. 1). In diesem Talabschnitt besitzt das Lechtal eine Breite von ca. $3,3 \mathrm{~km}$ unmittelbar nördlich der Jungendmoräne, die auf etwa 5,3 km talabwärts zunimmt.

Den geologischen Rahmen des Untersuchungsgebietes bildet das Vorland des hochwürmzeitlichen Maximalvorstoßes von Lech- und Ammerseegletscher im westlichen Alpenvorland. Im Südosten wird der untersuchte Lechtalabschnitt von der Jungmoränenlandschaft, im Nordosten vom lößbedeckten Altmoränengebiet des Loisachgletschers begrenzt. Den westlichen Talrahmen bilden Deckenschotter unterschiedlicher zeitlicher Stellung. An beiden Talflanken streichen lokal rutschungsanfällige Feinklastika der Oberen Süßwassermolasse aus. Unterhalb von Pitzling wird das Lechtal von der rißzeitlichen Hochterrasse begleitet, die mit den Altmoränen des Wertachgebiets verbunden ist. Als „Augsburger Hochterrasse“ erstreckt sie sich bis in das Stadtgebiet von Augsburg, wo sie von Lech und Wertach erodiert wurde.

Das Gefälle des Talgrundes nimmt von ca. 7,5 \% im Bereich des Übergangskegels zwischen Kinsau und Unterbergen auf bis zu $3 \%$ im Lechfeld bei Augsburg ab. Das relativ hohe Talgefälle und das alpine Abflussregime sind die wesentlichen Gründe für das natürliche flussmorphologische Erscheinungsbild eines stark verzweigten Flusses. 


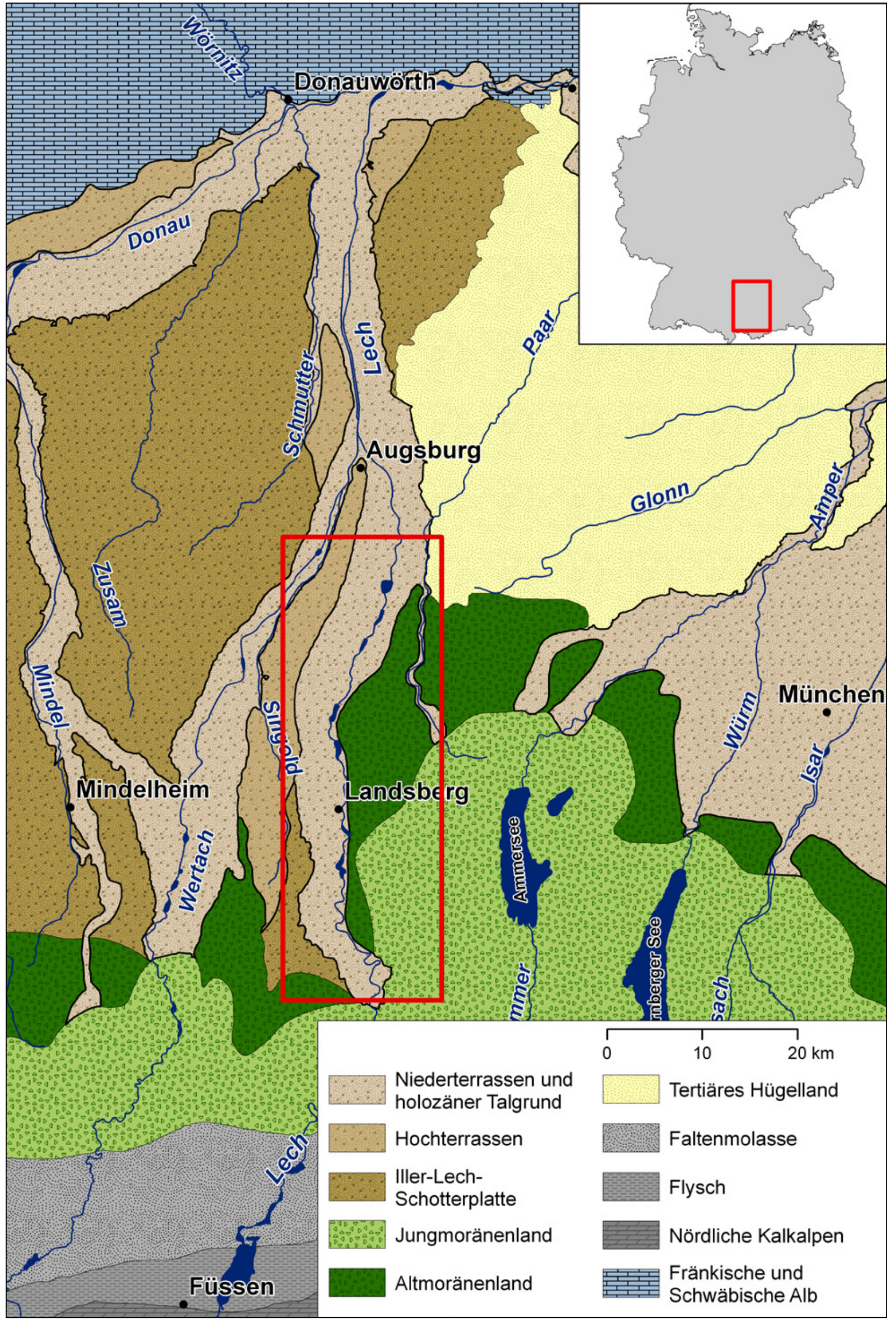

Abb. 1: Lage des Untersuchungsgebietes.

Fig. 1: Study area. 
Erst die neuzeitlichen flussbaulichen Korrektionsmaßnahmen (BAUER 1979) haben den Lech zunehmend auf ein durch Hochwasserdämme und verschiedene Uferverbauungen eingefasstes Flussbett eingeengt. Mit dem Bau von Staustufen an der Wende zum 20. Jahrhundert wurde der Lech letztendlich kanalartig verbaut. Heute regeln 24 Staudämme und Stauseen den Lechabfluss. Zeugnisse eines im Würmhoch- und -spätglazial verwilderten (braided river) und im Holozän stark verzweigten Flussbetts sind die innerhalb der Terrassenoberflächen heute noch erhaltenen zahlreichen Haupt- und Nebenarme des Lechs. Insofern bietet der Lech die Möglichkeit zur Untersuchung der jungquartären Talgeschichte an einem natürlicherweise auch unter warmzeitlichen Klimabedingungen stark verzweigten Flusslauf.

Der Fokus dieses Beitrages liegt auf der Darstellung neuer Befunde zur stratigraphischen Differenzierung, Lagerung und Altersstellung der im Untersuchungsgebiet erhaltenen jungquartären Lechterrassen.

\section{Forschungsstand}

Mit der jungquartären Terrassengliederung des Lechtals befassten sich erstmalig Troll (1925, ders. 1926) und KNAUER (1929), letzterer im Rahmen der Aufnahmen zur Geognostischen Karte Bayerns (Blatt München West; Maßstab 1:100 000) (Tab. 1). Dabei wurden, abgesehen von einer Alluvialzone entlang des Lechs, vier bis sieben weitere Terrassenstufen ausgegliedert und als würmzeitliche Bildungen beschrieben (Tab. 1). Es war BRUNNACKER (1959, ders. 1964), der im Raum Epfach erstmalig die Möglichkeit einer weitergehenden Differenzierung der holozänen Lechterrassen aufgezeigt hat. Er konnte dort neben der holozänen Talaue drei holozäne Lechterrassen, die beiden Lorenzbergstufen und eine römerzeitliche Talstufe, nachweisen. Diese erste Untergliederung des holozänen Talbodens des Lechs wurde dann von Diez (1968, ders. 1973) im Rahmen einer großmaßstäbigen Kartierung des Lechtals im Raum Landsberg weiter verfeinert. SchreIBER (1985) und Grottenthaler (1993, ders. 2009) folgen anschließend weitgehend der stratigraphischen Untergliederung der Lechterrassen von Diez (1968), wobei SCHrEIBER (1985) zudem innerhalb der würmzeitlichen Lechablagerungen schotterpetrographisch eine Isar-Loisach-Fazies von einer Iller-Lech-Schüttung abgrenzen konnte.

\section{Methodik}

Die stratigraphische Differenzierung der jungquartären Lechterrassen stützt sich vor allem auf ausgedehnte geomorphologische Kartierungen sowie zahlreiche Aufschlussaufnahmen der fluvialen Terrassenkörper, ihrer Deckschichten und Bodenbildungen. Böden und Deckschichten wurden zudem über eigene Handbohrungen erfasst. Auswertungen von Schichtenverzeichnissen von Bohrungen lieferten vor allem Informationen über die Mächtigkeiten der quartären Lechablagerungen. Historische Kartenwerke (Flurkarten der Bayerischen Uraufnahme 1808-1811, Renovationsaufnahmen von 1846) dienten der Rekonstruktion alter Flussläufe vor den Flusskorrektionen und waren sehr hilfreich bei der Abgrenzung der jüngsten Auen. Hoch- auflösende LIDAR-Daten (1 m Bodenauflösung), auf deren Basis 3D-Geländemodelle und Hangschattierungsmodelle errechnet wurden, ermöglichten eine feingliedrige Reliefdifferenzierung auch in schwer zugänglichem Gelände der großflächig bewaldeten Lechauen. Sämtliche Altersangaben erfolgen in konventionellen (nicht kalibrierten) ${ }^{14} \mathrm{C}$-Jahren vor 1950 (a BP). Dabei ist zu berücksichtigen, dass alle ${ }^{14} \mathrm{C}$-Alter von Schneckenschalen und Holzhäckseln aus Mergelschollen stammen. Da es sich dabei um umgelagertes Material handelt, geben die Datierungen das maximale Alter der entsprechenden Terrassen an.

\section{Die jungpleistozänen Lechterrassen im Einzelnen}

\subsection{Die Übergangsterrasse südlich Landsberg}

Die Übergangsterrasse ist die älteste jungpleistozäne Lechterrasse (Abb. 2a). Sie ist flächenhaft von bis zu einem Meter mächtigen sandstreifigen Lössablagerungen bedeckt und lediglich als schmale Terrassenleiste am östlichen Talrand südlich von Landsberg erhalten. Aufgrund ihrer Höhenlage im Tal, ca. $5 \mathrm{~m}$ über dem Niveau der Hauptniederterrasse (HNT), und ihrer Sandlössbedeckung ist sie älter als die hochglaziale, über Schmelzwasserbahnen mit den Jungendmoränenständen im Raum Hohenfurch verknüpfte HNT und jünger als die risszeitliche Hochterrasse des Lechs. Vermutlich entstand sie im Früh- oder Mittelwürm, könnte allerdings auch eine eemzeitliche Bildung sein.

\subsection{Die würmzeitlichen Niederterrassen}

Die würmzeitlichen Niederterrassen bestehen im Einzelnen aus folgenden stratigraphischen Untereinheiten (Tab. 2): Die lößfreie hochglaziale HNT und ihre proglazialen Teilfelder nehmen etwa $65 \%$ des südlichen Talgrunds ein. Diez (1968), Schreiber (1985) sowie Troll (1925, ders. 1926) weisen darauf hin, dass die HNT morphologisch mit dem Übergangskegel der äußersten Jungendmoränen des würmhochglazialen Lechgletschers bei Hohenfurch und die tiefer gelegene späthochglaziale "Stufe von Altenstadt“ mit dem Rückzugsstand des Lech- (Schongauer Zunge) bzw. Isar-Loisach-Gletschers (Ammersee-Zunge) bei Tannenberg bzw. St. Ottilien verknüpft ist. Zudem wird eine Verknüpfung der späthochglazialen „Stufe von SchongauPeiting“ mit dem Rückzugsstand bei Bernbeuern (TrOLL 1925) angenommen. Für die nächstjüngere „Stufe von Unterigling“ versuchte bereits Troll (1925) eine Anbindung an den Rückzugsstand bei Weilheim im Füssener Becken zu beschreiben. Neue Altersbelege (s. u.) weisen jedoch eine spätglaziale Alterstellung nach.

Talabwärts gehen die im Übergangskegel morphologisch klar abgesetzten Oberflächen der HNT und ihrer Teilfelder sukzessive in ein morphologisch nicht weiter differenzierbares Niederterrassenniveau über (Abb. 3). Südlich von Dornstetten (Abb. 3) unterschneidet die späthochglaziale „Stufe von Altenstadt“ das hochglaziale HNT-Niveau und ist talabwärts bis in den Raum Kaufering die älteste Niederterrassenfläche im Lechtal. Die späthochglaziale Stufe von Schongau-Peiting ist im Untersuchungsgebiet durch eine deutliche Terrassenkante von den älteren Lechterrassen 


\begin{tabular}{|c|c|c|c|c|c|c|c|c|c|c|c|c|c|c|c|c|c|c|c|}
\hline 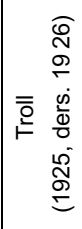 & \multicolumn{3}{|c|}{ 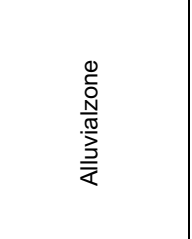 } & & & & & 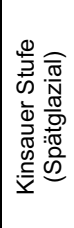 & 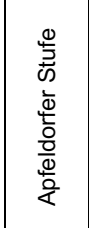 & & & & & 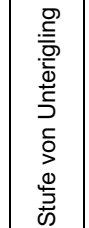 & 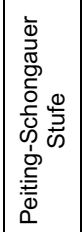 & 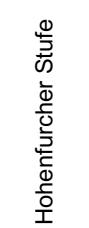 & 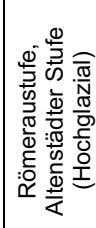 & & 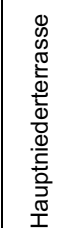 \\
\hline 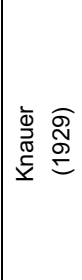 & \multicolumn{3}{|c|}{ 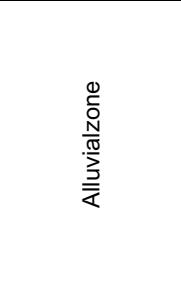 } & & & & & 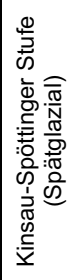 & 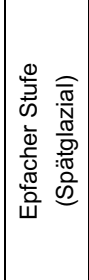 & & & & & & & & 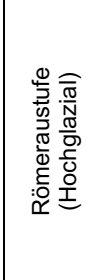 & & 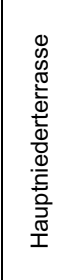 \\
\hline 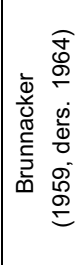 & \multicolumn{2}{|c|}{ 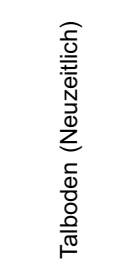 } & 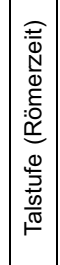 & & 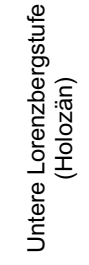 & 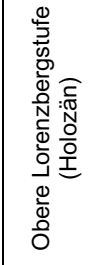 & & 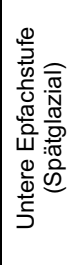 & 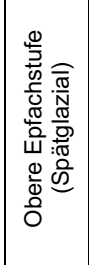 & & & & & & & & 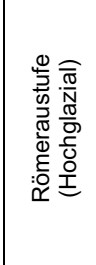 & & 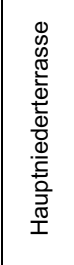 \\
\hline 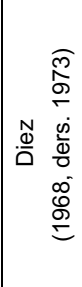 & 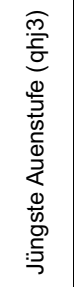 & 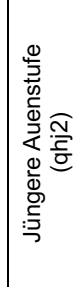 & 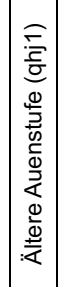 & & 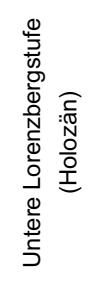 & 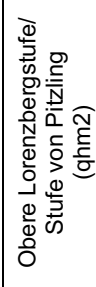 & 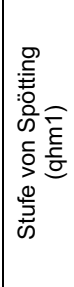 & 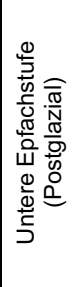 & 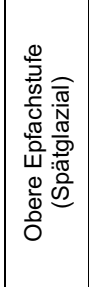 & 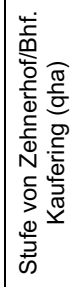 & & 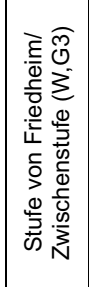 & & 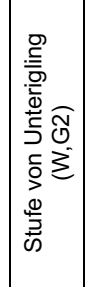 & 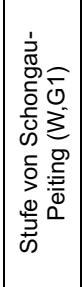 & 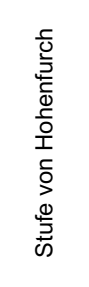 & 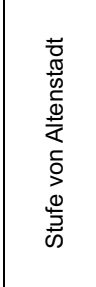 & 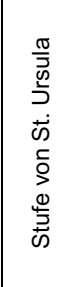 & 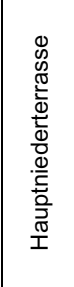 \\
\hline 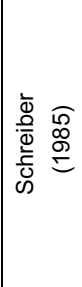 & 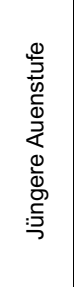 & 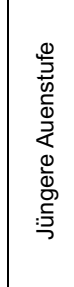 & 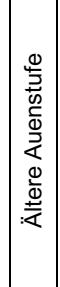 & & 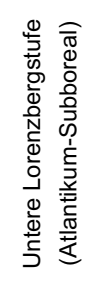 & 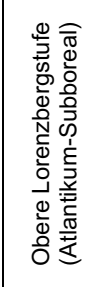 & & 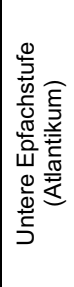 & 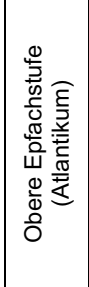 & 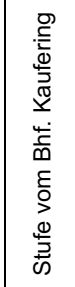 & 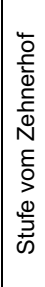 & 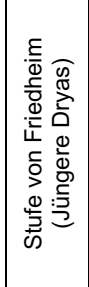 & 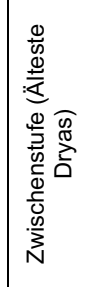 & 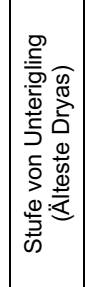 & 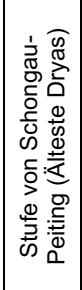 & 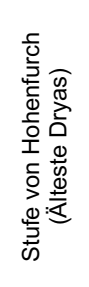 & 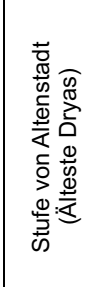 & 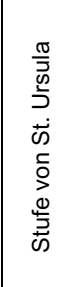 & 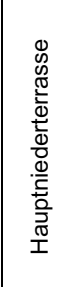 \\
\hline 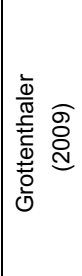 & 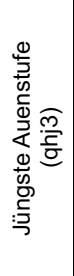 & 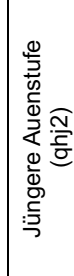 & 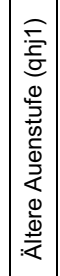 & & 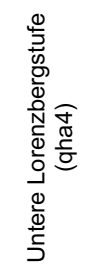 & 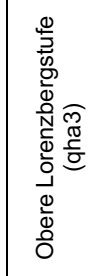 & & 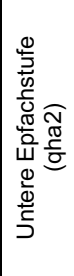 & 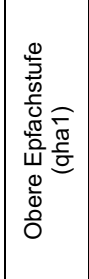 & & & & & & 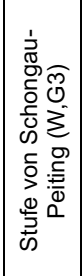 & 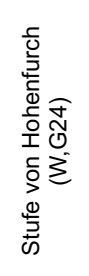 & 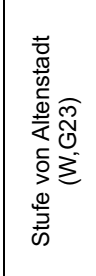 & 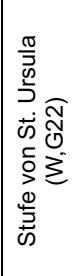 & 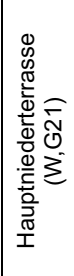 \\
\hline 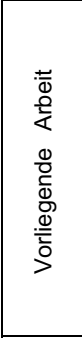 & 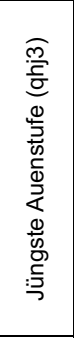 & 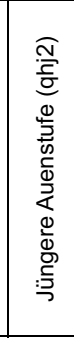 & 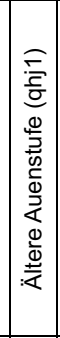 & 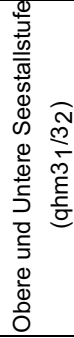 & 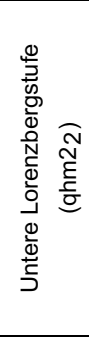 & 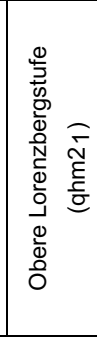 & 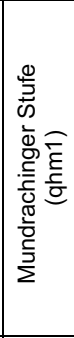 & 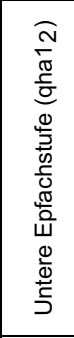 & 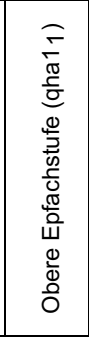 & & & 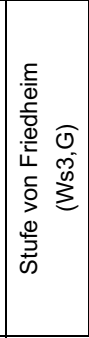 & 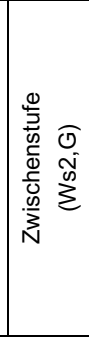 & 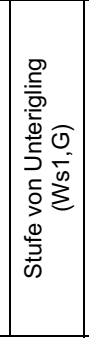 & 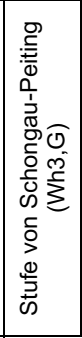 & & 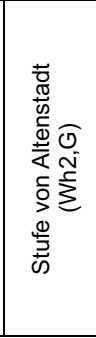 & & 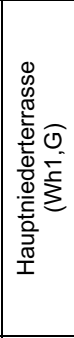 \\
\hline$\frac{\bar{d}}{\frac{1}{4}}$ & 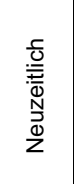 & 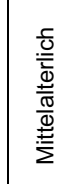 & 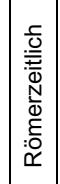 & & $\begin{array}{l}\overline{\bar{\Xi}} \\
\overline{0} \\
\overline{0} \\
\bar{\Xi} \\
\bar{\rho}\end{array}$ & & 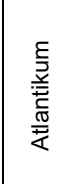 & & 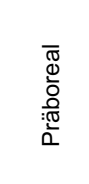 & & & & 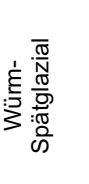 & & & $\xi$ & & & 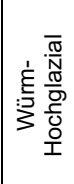 \\
\hline
\end{tabular}




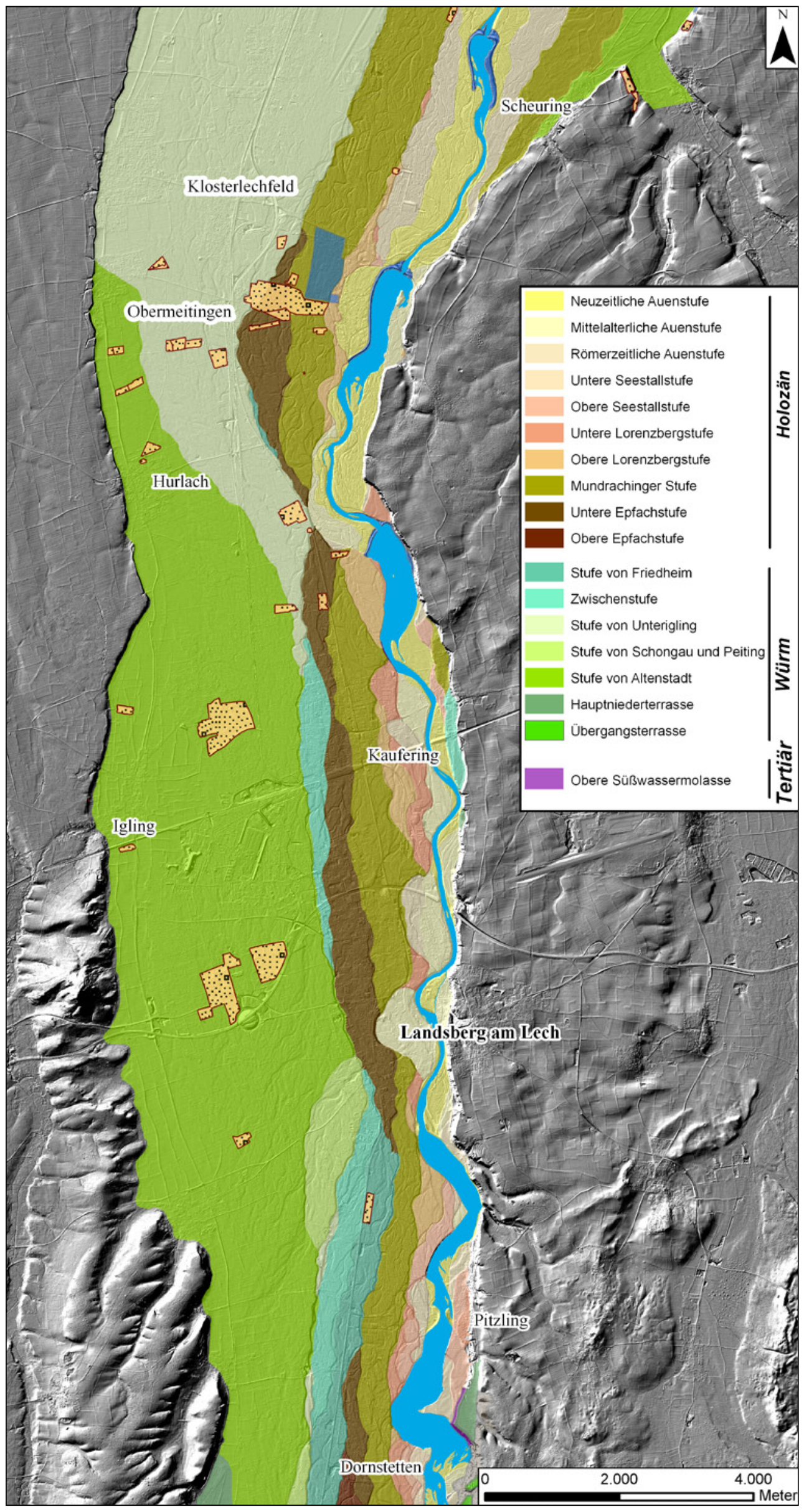

Abb. 2a: fungquartärer Talboden zwischen Kinsau und Landsberg. Fig. 2a: Late Quaternary valley bottom between Kinsau and Landsberg. 


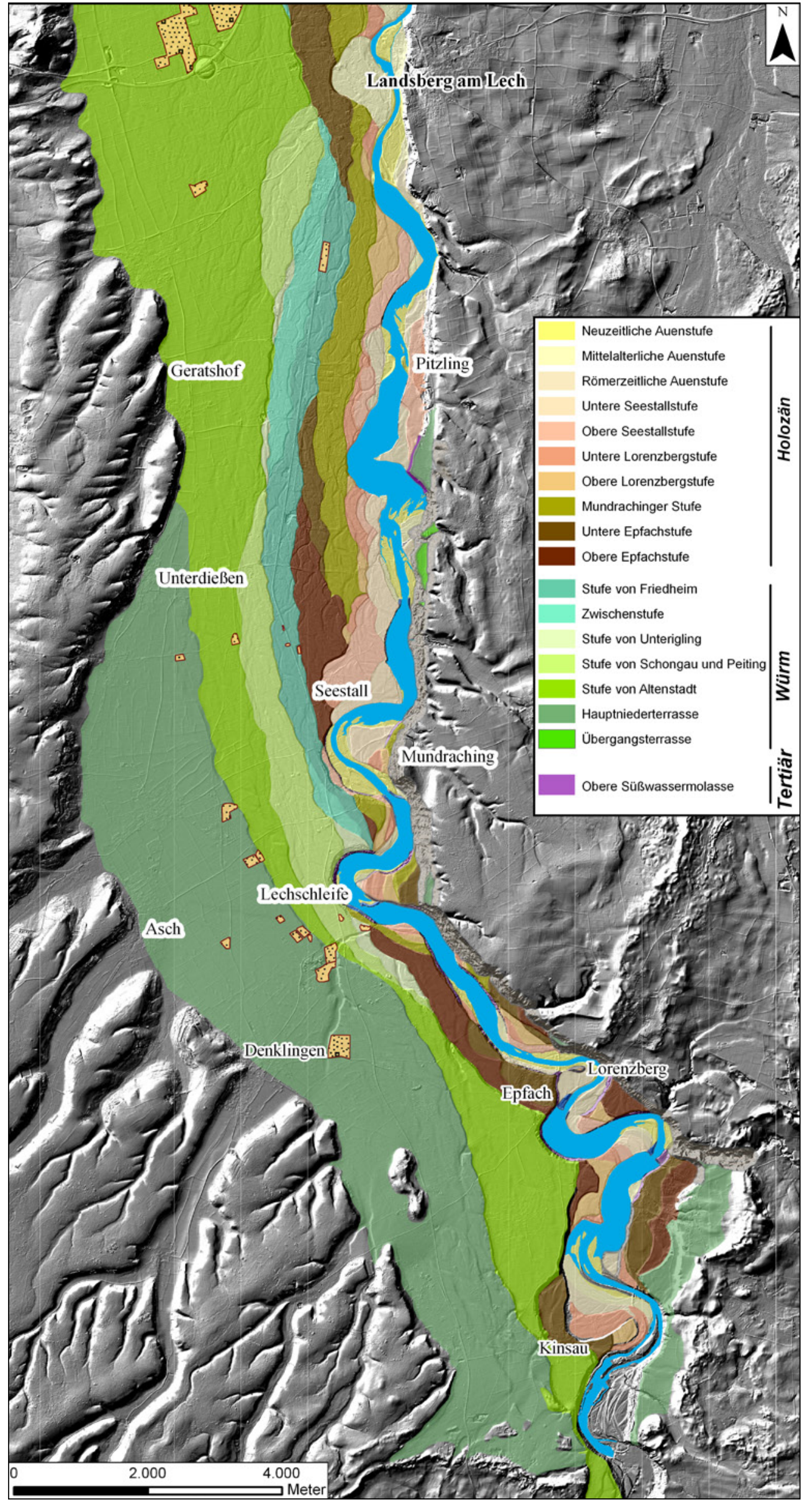

Abb. 2b: fungquartärer Talboden zwischen Landsberg und Klosterlechfeld.

Fig. 2b: Late Quaternary valley bottom between Landsberg and Klosterlechfeld. 
abgesetzt und am südlichen Stadtrand von Landsberg vom altholozänen Lech ausgeräumt worden.

Die Kieskörper der HNT und ihre Teilfelder wurden während des Würm-Hochglazials von einem verwilderten (braided river) und relativ breiten Lechfluss aufgeschüttet. Entsprechende Rinnenstrukturen ehemaliger Flussarme prägen noch heute deren Oberflächen. Bereichsweise wurden die Terrassenoberflächen noch im ausgehenden Würm unter periglazialen Klimabedingungen von Trockentälern zerschnitten. Kiesgruben zeigen, als Indiz für einen hochenergetischen verwilderten (braided river) Fluss, ein vertikal aufgehöhtes, horizontal- und troggeschichtetes Sedimentationsbild grobklastischer Sedimente. Es gibt keine Hinweise, die auf eine längere interstadiale Unterbrechung der vertikalen Schotterakkumulationen hindeuten. Anhand von Schotteranalysen konnte ScHREIBER (1985) jedoch aufzeigen, dass insbesondere die Kiese der Niederterrassen bereichsweise in eine Lech- bzw. Loisachfazies unterteilt werden können.

Die relativ flachgründigen Bodenentwicklungen auf den Würmterrassen sind durch ackerbauliche Nutzung stark überprägt. Generell nimmt die Mächtigkeit der schluffig-sandigen Deckschichten von der HNT zu den jüngeren Teilfeldern ab. An einzelnen Standorten sind, wie bereits von DiEz (1968) und WiLKE (1975) beschrieben, als maximale Bodenentwicklungen statt ackerbaulich geprägter „Kulto-Rendzinen“, Parabraunerden und Braunerden erhalten.

\subsubsection{Die Hauptniederterrasse}

Der Übergangskegel des Maximalstandes des Lechgletschers setzt sich im weiteren Talverlauf nördlich von Hohenfurch in der hochglazialen HNT fort (Abb. 2a, Abb. 3). Diese liegt bei Kinsau in etwa $73 \mathrm{~m}$ und ca. $14 \mathrm{~km}$ weiter im Norden bei Unterdießen noch in etwa $33 \mathrm{~m}$ Höhe über dem aktuellen Lechlauf. Auf der $5 \mathrm{~km}$ langen Talstrecke nördlich Kinsau erreicht die HNT eine Breite von etwa $1,5 \mathrm{~km}$ und ein Talgefälle von 7,5\%. In diesem Talabschnitt sind in der Oberflächenmorphologie der HNT kleinere, nicht durchhaltende Geländestufen erhalten. Die Oberflächen dieser proglazialen Teilfelder dachen talabwärts auf ein einheitliches HNT-Niveau ab. Dieses weist zwischen Asch und Unterdießen nur noch ein Oberflächengefälle von etwa $5,4 \%$ auf. Gleichzeitig nimmt die Breite der HNT auf etwa $2 \mathrm{~km}$ zu. Die Kiese der HNT erreichen im Bereich des

Tab. 2: Altersstellung der jungquartären Lechterrassen im Überblick.

Tab. 2: Chronology of Late Quaternary Lech terraces.

\begin{tabular}{|c|c|c|c|}
\hline Terrassenstufen & Quartärmächtigkeiten & Altersstellung & Altersbelege \\
\hline Jüngste Auenstufe & bis zu $10 \mathrm{~m}$ & Neuzeitlich & Historische Karten \\
\hline Jüngere Auenstufe & bis zu $10 \mathrm{~m}$ & Mittelalterlich & Archäologische Daten \\
\hline Ältere Auenstufe & bis zu $10 \mathrm{~m}$ & Römerzeitlich & Archäologische Daten \\
\hline $\begin{array}{l}\text { Obere und Untere } \\
\text { Seestallstufe }\end{array}$ & 2 bis $7 \mathrm{~m}$ & \multirow{2}{*}{ Subboreal } & --- \\
\hline $\begin{array}{l}\text { Obere und Untere } \\
\text { Lorenzbergstufe }\end{array}$ & 2 bis $6 m$ & & --- \\
\hline Mundrachinger Stufe & 6 bis $17 \mathrm{~m}$ & Atlantikum & $\begin{array}{l}5720 \pm 40{ }^{14} \mathrm{C} \text { BP (Holzhäcksel in Humusscholle) } \\
5900 \pm 40{ }^{14} \mathrm{C} \text { BP (Holzhäcksel in Humusscholle) } \\
5855 \pm 205{ }^{14} \mathrm{C} \text { BP (Holzhäcksel in Humusscholle) } \\
\text { (NIEDERSÄCHSISCHES LANDESAMT FÜR BODENFORSCHUNG) }\end{array}$ \\
\hline $\begin{array}{l}\text { Obere und Untere } \\
\text { Epfachstufe }\end{array}$ & 8 bis $23 m$ & Präboreal & $9950 \pm 50{ }^{14} \mathrm{C}$ BP (Schneckenschale in Lehmscholle) \\
\hline $\begin{array}{l}\text { Zwischenstufe und } \\
\text { Stufe von Friedheim }\end{array}$ & 18 bis $24 \mathrm{~m}$ & \multirow{2}{*}{ Würm-Spätglazial } & $10120 \pm 60{ }^{14} \mathrm{C}$ BP (Schneckenschale in Lehmscholle) \\
\hline Stufe von Unterigling & 13 bis $26 \mathrm{~m}$ & & $\begin{array}{l}11760 \pm 50{ }^{14} \mathrm{C} \text { BP (Schneckenschale in Lehmscholle) } \\
12610 \pm 50{ }^{14} \mathrm{C} \text { BP (Schneckenschale in Lehmscholle) }\end{array}$ \\
\hline $\begin{array}{l}\text { Stufe von Schongau- } \\
\text { Peiting }\end{array}$ & 16 bis $31 \mathrm{~m}$ & \multirow{2}{*}{$\begin{array}{l}\text { Würm- } \\
\text { Späthochglazial }\end{array}$} & Anschluss an Jungendmoräne („Bernbeuernstadium“) \\
\hline Stufe von Altenstadt & 15 bis $27 \mathrm{~m}$ & & Anschluss an Jungendmoräne („Tannenberg“, „St. Ottilien“) \\
\hline Hauptniederterrasse & 15 bis $68 \mathrm{~m}$ & Würm-Hochglazial & $\begin{array}{l}\text { Anschluss an Jungendmoräne („Hohenfurch“) } \\
\text { OSL-Datierung fluvialer Sande } 22030 \pm 2030 \text { BP }\end{array}$ \\
\hline
\end{tabular}




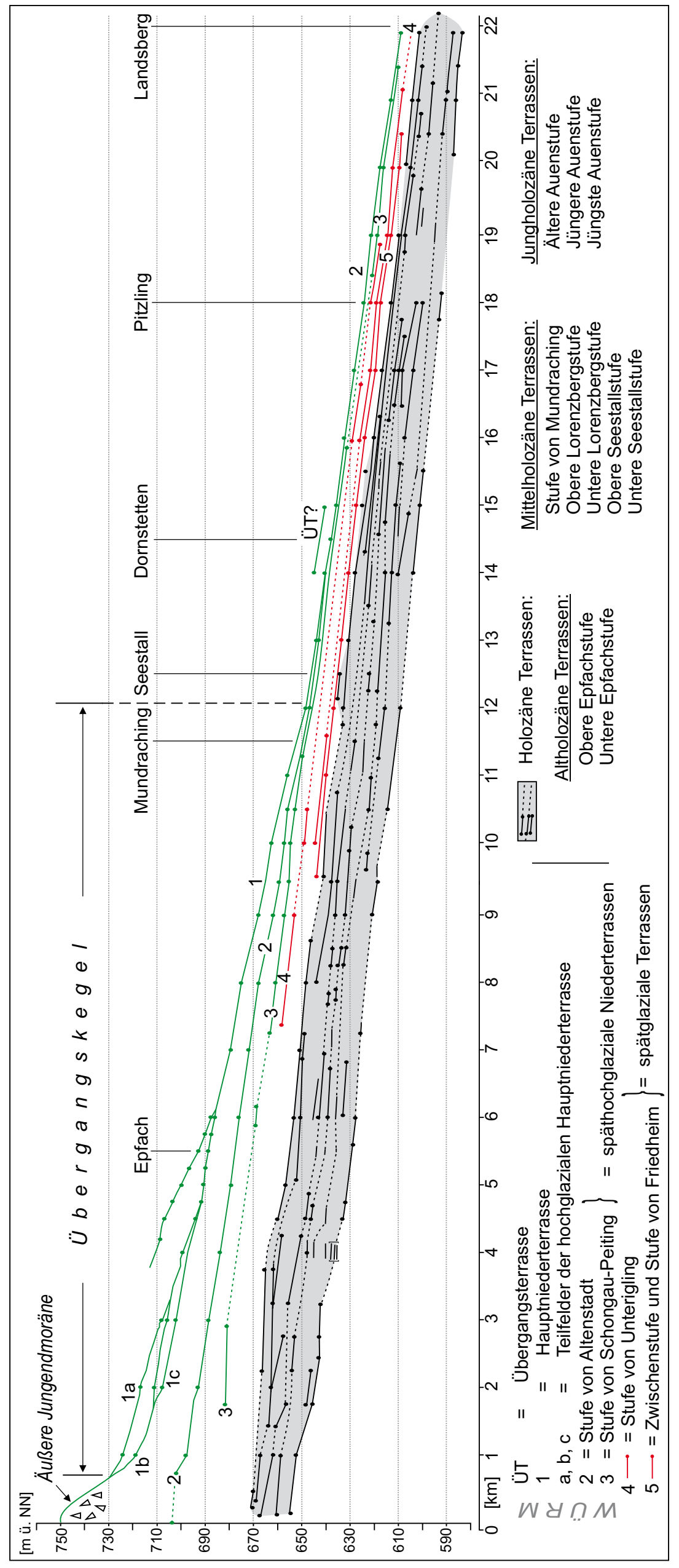

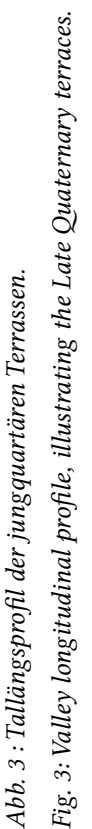


Übergangskegels Mächtigkeiten von über $68 \mathrm{~m}$, die talabwärts auf $35 \mathrm{~m}$ im Raum Kinsau und auf $15 \mathrm{~m}$ im Raum Unterdießen abnehmen. Der Anteil des HNT-Kieskörpers an der gesamten Mächtigkeit der Quartärsedimente ist nur unzureichend bekannt. Im Bereich des Übergangskegels existieren nach einer Aufschlussbeobachtung südlich von Kinsau Mindestmächtigkeiten von mehr als $21 \mathrm{~m}$.

Im Untersuchungsgebiet sind auf der HNT weitflächig schluffig-sandige Deckschichten von 50 bis $100 \mathrm{~cm}$ Mächtigkeit verbreitet, die vermutlich Flugsande darstellen. Auf ihnen sind Parabraunerden mit Entwicklungstiefen von bis zu $50 \mathrm{~cm}$ unter Flur verbreitet (siehe auch DiEz 1968, WiLKE 1975). Aufgrund der morphologischen Verknüpfung der HNT und ihrer proglazialen Teilfelder (Abb. 3) mit den äußeren Jungendmoränen des Lech- und des Loisachgletschers bei Hohenfurch (Sachsenrieder bzw. Reichlinger Stand) ist eine Bildungszeit im Hochglazial bis vor ca. 18000 a BP gesichert (Tab. 2).

\subsubsection{Die Stufe von Altenstadt}

Die Stufe von Altenstadt, die der „Römeraustufe“ bzw. der „Altenstädter Stufe“ von Troll (1925, ders. 1926) (Tab. 1) entspricht, wird von verschiedenen Bearbeitern (Grottenthaler 2009, Diez 1968, Knauer 1929, Troll 1925, ders. 1926) mit dem ersten Rückzugsstadium des Lechgletschers verknüpft („Tannenberger Stand“ sensu Troll 1926). KNAUER (1929) sieht eine Verknüpfung dieses ältesten Teilfeldes der Niederterrasse mit einem Endmoränenkranz nördlich von Burggen, was dem „Tannenberger Stand" von Troll (1925) entspricht.

Nach dem Durchbruch durch die Jungendmoränen ist die Stufe von Altenstadt ab Kinsau im gesamten Untersuchungsgebiet auf der westlichen Talseite des Lechs morphologisch erhalten. Im Talraum oberhalb von Unterdießen ist sie durch einen mehrere Meter hohen Stufenrand morphologisch deutlich von der höheren HNT abgesetzt. Allerdings gleichen sich die Oberflächenniveaus beider Terrassenstufen infolge ihrer unterschiedlichen Oberflächengefälle (HNT ca. 5,4\%o, Stufe von Altenstadt ca. 5,0 \%o) im Raum Unterdießen zunehmend an. Informationen über die Tiefenlage ihrer Terrassenbasis liegen nicht vor. Im Lechtal unterhalb von Unterdießen ist die Stufe von Altenstadt die dominante Niederterrassenfläche, ehe sie zwischen Kaufering und Hurlach von der spätglazialen Stufe von Unterigling ausgeräumt wird.

Unterhalb von Scheuring ist erstmals auch auf der östlichen Seite des Lechs eine Niederterrasse erhalten, die von ihrer Höhenlage im Tal wahrscheinlich der Stufe von Altenstadt entspricht oder etwas älter ist. Auf diese Terrassenleiste mündet von Südosten das würmzeitliche Schmelzwassertal des „Verlorenen Baches“ ein, das nach Krause (mündl. Mitteilung) mit dem ersten Rückzugsstand des Ammerseegletschers verbunden werden kann.

Der quartäre Kieskörper der Stufe von Altenstadt besitzt im südlichen Untersuchungsgebiet Mächtigkeiten von ca. $27 \mathrm{~m}$, die talabwärts auf ca. $15 \mathrm{~m}$ bei Kaufering abnehmen (Tab. 2). Welchen Anteil daran eventuell ältere Lechablagerungen haben, ist nicht bekannt.

Die Stufe von Altenstadt besitzt im südlichen Untersuchungsgebiet ähnlich der HNT häufig eine sandige Deck- schicht aus vermutlich Flugsanden, die allerdings nur Mächtigkeiten von bis zu $60 \mathrm{~cm}$ erreichen.

Es liegen keine neuen chronostratigraphischen Befunde zur Altersstellung der Stufe von Altenstadt vor. Nach den bisherigen Bearbeitern (s. o.) ist sie mit dem 1. Rückzugsstand des Lechgletschers zu verknüpfen und damit im späten Würmhochglazial entstanden. Sie ist allerdings nicht die älteste Terrassenstufe aus der frühen Abschmelzzeit des Würmhochglazials. Nach RATHJENS (1951) sowie Grottenthaler (2009) ist die Stufe von St. Ursula, die außerhalb des Untersuchungsgebietes bei Schongau innerhalb der äußeren Jungendmoränenzüge erhalten ist, noch etwas älter.

\subsubsection{Die Stufe von Schongau-Peiting}

Die Stufe von Schongau-Peiting wurde erstmalig von TroLL (1925) südlich des Arbeitsgebietes im „Peitinger Trockental“ und auf dem Umlaufberg von Schongau beschrieben (Tab. 1). Nach Diez (1968) entstand diese Stufe im Hochglazial, zu einer Zeit, als der Lechgletscher etwa die Hälfte des ehemals eisbedeckten Vorlandes freigegeben hatte.

Im Untersuchungsgebiet setzt die Stufe von SchongauPeiting erst unterhalb von Epfach ein und erstreckt sich, mit kurzen Unterbrechungen, bis Landsberg (Abb. 2a). Morphologisch ist sie durch eine deutliche, im südlichen Untersuchungsgebiet wenige Meter hohe Böschung von der Stufe von Altenstadt abgesetzt und besitzt ein Oberflächengefälle von lediglich $3,7 \%$. Da die zum westlichen Talrand hin angrenzende Stufe von Altenstadt ein höheres Oberflächengefälle besitzt, nähern sich die Oberflächen beider Terrassen zwischen Denklingen und Unterdießen bis auf wenige Dezimeter an. Im Gegensatz zu den älteren Niederterrassenstufen treten auf der Stufe von SchongauPeiting sehr deutlich ausprägte Flussrinnensysteme (channels) auf, die meist parallel zum Außenrand der Terrasse verlaufen. Sie sind wenige Dezimeter tief und verfügen über keine feinklastischen Füllungen.

Die Gesamtmächtigkeit der Quartärschotter schwankt zwischen 16 bis $31 \mathrm{~m}$ (Tab. 2), wobei die Tiefenlage der Terrassenbasis nicht bekannt ist. Aufschlüsse, die Aussagen über das Schichtungsbild des Terrassenkörpers ermöglichen, existieren nicht. Allerdings ist aufgrund des verwilderten Gerinnebettmusters auf der Terrassenoberfläche von einem ähnlichen Sedimentationsbild wie bei den älteren Niederterrassen auszugehen. Auch der Grad der maximalen Bodenentwicklung in Form von Parabraunerden mit durchschnittlichen Mächtigkeiten von 40 bis $50 \mathrm{~cm}$ (siehe auch DiEz 1968) liefert kein weiteres Unterscheidungskriterium zu den älteren würmzeitlichen Terrassenstufen.

Die Stufe von Schongau-Peiting ist jünger als die Stufe von Altenstadt (und die im Untersuchungsgebiet nicht mehr nachweisbare Stufe von Hohenfurch) und kann nach Troll (1925, ders. 1926) mit den Jungendmoränen bei Bernbeuren verknüpft werden. Insofern sollte sie im späten Würmhochglazial entstanden sein.

\subsubsection{Die Stufe von Unterigling}

Die Stufe von Unterigling wurde erstmalig von Troll (1925) mit den Jungendmoränen des Lechgletschers am Nor- 
drand des Füssener Beckens und des Ammerseegletschers bei Weilheim verknüpft (Tab. 1). Diez (1968) und SchreIBER (1985) folgten der Alterseinstufung von Troll (1925).

Im Untersuchungsgebiet sind bis Landsberg lediglich vier kleinere Terrassenreste der Stufe von Unterigling erhalten. Wegen ihres geringeren Gefälles konvergieren deren Oberflächen talabwärts zunehmend mit der Stufe von Altenstadt. Dadurch verringert sich der Höhenunterschied zwischen beiden Terrassenstufen von etwa $2 \mathrm{~m}$ im Raum Erpfting auf nur wenige Dezimeter am südwestlichen Stadtrand von Landsberg.

Erstmalig großflächig erhalten ist die Stufe von Unterigling etwa 9,5 km nördlich von Landsberg. Auf Kosten der Stufe von Altenstadt gewinnt sie in etwa bei Kaufering zunehmend an Ausdehnung und dominiert ab Hurlach, wo die Stufe von Altenstadt vollständig erodiert ist, den jungpleistozänen Talgrund. Auch die Oberfläche der Stufe von Unterigling ist von zahlreichen ehemaligen Flussrinnen geprägt.

Die Mächtigkeit des quartären Kieskörpers im Bereich dieser Terrasse schwankt zwischen 13 und $26 \mathrm{~m}$. Die Tiefenlage ihrer Terrassenbasis ist unbekannt. Aufschlüsse nördlich von Kaufering zeigen einen mehrere Meter mächtigen, von einer meist schluffig-sandigen Matrix gestützten, horizontal- und troggeschichteten V-Schotterkörper (sensu Schirmer 1983). Anders als bei den hochglazialen Flussschottern sind in den hangenden Flusskiesen der Stufe von Unterigling Mergelschollen mit Resten von Schneckenschalen eingelagert.

Die maximale Bodenentwicklung in Form von Parabraunerden (siehe auch DiEz 1968) entspricht weitgehend der auf den älteren Niederterrassen.

Entgegen der oben beschriebenen Moränenverknüpfungen von Troll (1925), Diez (1968) und Schreiber (1985) belegen ${ }^{14} \mathrm{C}$-AMS-Datierungen an Fragmenten von zwei in ihrem Kieskörper in $2 \mathrm{~m}$ Tiefe eingelagerten Schneckenschalen (11 $760 \pm 50$ a BP; $12610 \pm 50$ a BP) eine spätglaziale Alterstellung der Stufe von Unterigling.

\subsubsection{Die Zwischenstufe und die Stufe von Friedheim}

Die Zwischenstufe und die Stufe von Friedheim wurden erstmalig von DiEz (1968) beschrieben. Beide Terrassen sind im südlichen Kartengebiet erstmals nördlich der Lechschleife (Abb. 2a) erhalten. Sie bilden im weiteren Talverlauf zwei ausgedehnte Terrassenflächen, die bei Landsberg vom jungholozänen Mäanderbogen des Lechs ausgeräumt wurden. Unterhalb von Landsberg (Abb. 2b) ist die Stufe von Friedheim nur noch als schmale Terrassenleiste östlich von Hurlach erhalten. Dagegen erstreckt sich die Zwischenstufe bis Kaufering, wo sie auch östlich des Lechs als schmaler Terrassenrest angelegt ist.

Das durchschnittliche Oberflächengefälle beider Terrassen liegt bei 3,4\%o. Es ähnelt damit dem Gefälle der Stufe von Schongau-Peiting, ist aber deutlich niedriger als das der hochglazialen Niederterrassenfelder (Abb. 3). Von der Höhenlage ihrer Terrassenoberflächen sind beide Stufen sowohl von den hochglazialen Niederterrassen als auch von den holozänen Lechterrassen deutlich abgesetzt. Beide Stufen sind durch eine wenige Dezimeter bis maximal $1,5 \mathrm{~m}$ hohe Terrassenkante voneinander getrennt. Ebenso wie die Stufe von Unterigling besitzen auch diese beiden Stufen mehr oder minder anthropogen gekappte Braunerden.

Die Quartärbasis liegt mit 18 bis $23 \mathrm{~m} \mathrm{u}$. GOK in einer ähnlichen Tiefenlage wie bei der Stufe von Unterigling. Die Basis ihrer Terrassenkörper ist nicht bekannt. Informationen über das Schichtungsbild der Stufe von Friedheim lieferte ein Bauaufschluss an der neuen Trassenführung der B17 am südlichen Stadtrand von Landsberg. Ähnlich dem Kieskörper der Stufe von Unterigling sind in dem hangenden, 4 bis $5 \mathrm{~m}$ mächtigen, horizontal- und troggeschichteten Schotterkörper (ScHIRMER 1983) Mergelschollen mit Resten von Schneckenschalen eingelagert.

Die ${ }^{14} \mathrm{C}$-AMS-Datierungen an Fragmenten von Schneckenschalen, die aus einer Mergelscholle aus $3 \mathrm{~m}$ Tiefe unter Geländeoberfläche stammen, ergab ein Alter von $10120 \pm 60$ a BP. Da beide Stufen älter als die präborealen Epfachstufen (Tab. 2) sind, ist ihre Bildungszeit in das ausgehende Spätglazial zu stellen.

\section{Die holozänen Lechterrassen}

Auch im Holozän hatte der Lech das Bestreben sein im Hochglazial stark überhöhtes Gefälle auszugleichen. Im Zuge einer anhaltenden sukzessiven Eintiefung seiner Flussbettsohle entstanden mindestens 10 treppenartig angeordnete Terrassenniveaus (Tab. 2), die aber in keinem Talabschnitt in vollständiger Abfolge erhalten sind. Aufgrund der unterschiedlichen Höhenlage ihrer Terrassenoberflächen ist es möglich, auch kleinere Terrassenreste morphostratigraphisch einzuordnen. Die absolute Alterseinstufung dieser holozänen Terrassensequenz stützt sich bei den jüngsten Auenterrassen vor allem auf historische Flurkarten und wenige Bodendenkmäler sowie bei den beiden Epfachstufen und der Mundrachinger Stufe auf ${ }^{14} \mathrm{C}$-Daten.

Die nur wenige Meter mächtigen holozänen Kieskörper liegen auf würmzeitlichen Schmelzwasserschottern und sind häufig durch eine Blocklage mit eingelagerten Lehm-/ Humusschollen von diesen abgesetzt. Im Schichtungsbild ihrer Kieskörper dominieren Horizontal- und Trogschichtungen und belegen eine Ablagerung durch einen stark verzweigten Lechlauf, wobei mit Ausbildung der Unteren Epfachstufe erstmalig mäandergeformte Außenränder der Terrassen auftreten. An Bodenentwicklungen dominieren auf den alt- und mittelholozänen Terrassen Braunerden und (Para)rendzinen. Die jungholozänen Terrassen sind durch ihre bis zu $2 \mathrm{~m}$ mächtigen Flussmergeldecken und die wenig entwickelten Auenrendzinen von den älteren Terrassen des Lechs deutlich abgesetzt.

\subsection{Die Obere und Untere Epfachstufe}

Die von Troll (1925) noch als Kinsauer- und Apfeldorfer Stufe bezeichneten Lechterrassen wurden später von BRUNNACKER (1964) und auch von DiEz (1968) nach der auf ihnen liegenden Ortschaft Epfach benannt. Der westliche Teil des Ortes befindet sich auf der Oberen Epfachstufe, der östliche auf der etwa 1,5 m niedrigeren Unteren Epfachstufe.

Beide Epfachstufen treten erstmalig südlich des würmzeitlichen Jungendmoränengürtels, außerhalb des Untersuchungsgebietes, als kleinere Terrassenreste auf (Grottenthaler 2009). Am locus typicus bei Epfach 
bilden die beiden Terrassen westlich des Lechs einen etwa $400 \mathrm{~m}$ schmalen und 3,7 km langen Terrassenstreifen. Zwischen Landsberg und der Staustufe 18 bei Kaufering nimmt die Untere Epfachstufe große Areale westlich der Bundesstraße B17 ein (Abb. 2a, Abb. 2b), im Stadtgebiet von Landsberg bildet sie das morphologisch vermittelnde Element zwischen der späthochwürmzeitlichen Stufe von Altenstadt und der mittelalterlichen Jüngeren Auenstufe. Talabwärts setzt die Untere Epfachstufe erneut nördlich der Staustufe 18 bei Kaufering ein und bildet einen ca. 4,2 km langen Terrassenstreifen. Das Oberflächengefälle der beiden Epfachstufen beträgt lediglich 2,7 \%o bis 3,0 \%o und ist damit geringer als bei den älteren Lechterrassen (Abb. 3).

Die Quartärmächtigkeiten schwanken im Bereich der Epfachstufen zwischen 8 und 23 m, wobei nach Aufschlussbeobachtungen deren Kieskörper lediglich Mächtigkeiten von 2 bis $4 \mathrm{~m}$ besitzen. Wie bereits von Diez (1968) beschrieben, unterscheiden sich beide Epfachstufen durch ihre wenig entwickelten 25 bis $30 \mathrm{~cm}$ mächtigen Braunerden von den älteren Lechterrassen und deren Parabraunerden.

Die ${ }^{14} \mathrm{C}$-Datierung einer Schneckenschale aus einer Lehmscholle in 1,75 m Tiefe im Kieskörper der Unteren Epfachstufe nördlich von Kaufering ergab ein Alter von $9950 \pm 50$ a BP. Während die Untere Epfachstufe im Präboreal entstand, könnte die Obere Epfachstufe ebenfalls eine präboreale, allerdings auch noch eine jüngerdryaszeitliche Terrasse sein.

\subsection{Die Mundrachinger Stufe}

Die Mundrachinger Stufe wurde im Rahmen dieser Untersuchungen erstmalig ausgegliedert. Die von DiEz (1968) als Stufe von Spötting, in einzelnen Bereichen auch als Stufe vom Bahnhof Kaufering bezeichnete Terrasse bildet im Untersuchungsgebiet die flächenmäßig am weitesten verbreitete holozäne Terrassenstufe. Der locus typicus ist der Ort Mundraching, der zu großen Teilen auf dieser Terrasse liegt. Von dort setzt sich die Mundrachinger Stufe über 7,8 km talabwärts bis zum hochmittelalterlichen Terrassenniveau im Zentrum von Landsberg fort (Abb. 2a). Weiter talabwärts ist sie mit einer durchschnittlichen Breite von etwa $500 \mathrm{~m}$ bis zur Lechstaustufe 18 bei Kaufering erhalten (Abb. 2b), wo sie im Jungholozän ausgeräumt wurde. Unterhalb Kaufering flankiert die Terrasse die Lechaue zunächst nur auf der westlichen Talseite. Etwa $750 \mathrm{~m}$ südwestlich von Scheuring ist sie dann auch östlich des Lechs erhalten.

Die Mundrachinger Stufe weist ein Oberflächengefälle auf, das im Raum südlich von Landsberg bei etwa 3,3\%o liegt und talabwärts auf 3,1\%o abnimmt. Ihre Oberfläche prägen häufig zahlreiche zum Teil mit feinklastischen Sedimenten verfüllte Flussrinnen eines verzweigten Lechlaufs, die bis zu 1,5 m tief sein können. Auf dem Terrassenkörper sind humusreiche Pararendzinen entwickelt, wodurch sich diese Terrasse pedologisch von den älteren Lechterrassen unterscheidet. Die Quartärmächtigkeiten im Bereich der Mundrachinger Stufe schwanken zwischen 6 bis $17 \mathrm{~m}$. Nach Aufschlussbeobachtungen im Raum Obermeitingen besitzt ihr Kieskörper eine mittlere Mächtigkeit von etwa 4 bis $5 \mathrm{~m}$ und wird von älteren Lechschottern unbekannter Zeitstellung unterlagert.

Der horizontal- und troggeschichtete Kieskörper der Mundrachinger Stufe enthält vor allem an der Basis zahlreiche Lehmschollen (Abb. 4), die teilweise Schneckenschalen und organische Makroreste führen. Die ${ }^{14} \mathrm{C}$-Datierung von Pflanzenhäcksel aus einer Mergelscholle in ca. $3 \mathrm{~m}$ Tiefe unter Geländeoberfläche, die von Gerhard Doppler (Bay-

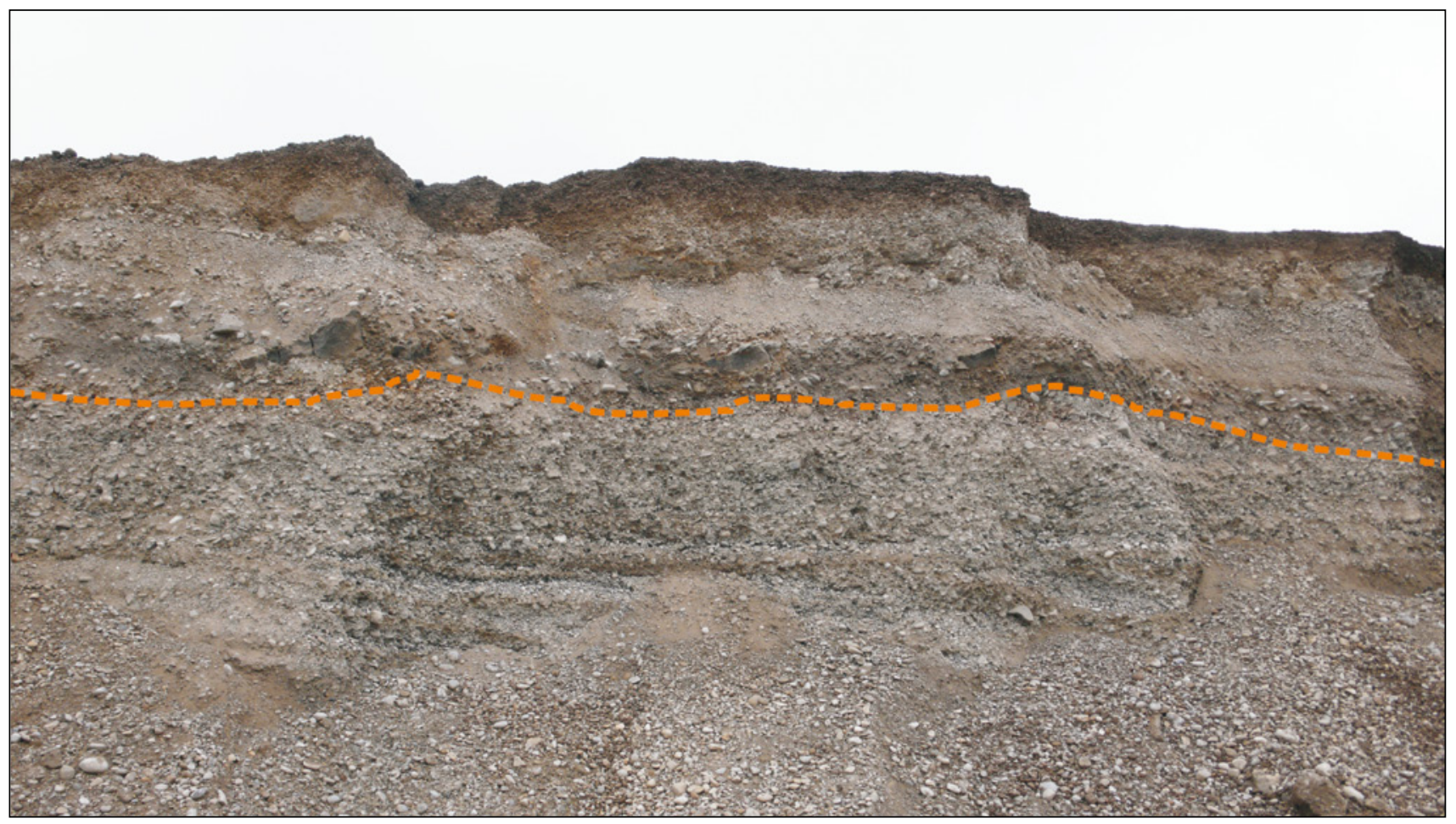

Abb. 4: Mittelholozäne Lechablagerungen der Stufe von Mundraching im Hangenden und würmzeitliche Niederterrassenschotter im Liegenden (Foto: B. Gesslein 2009).

Fig. 4: Middle Holocene Lech river deposits of the Stufe von Mundraching in the overlying strata and Würmian gravel in the underlying strata (Photo: B. Gesslein 2009). 
erisches Landesamt für Umwelt) in einer Kiesgrube bei Obermeitingen geborgen wurde, ergab ein ${ }^{14} \mathrm{C}$-Alter von $5855 \pm 205$ a BP (Tab. 2). Etwa 600 m weiter talabwärts konnten aus einer in der gleichen Terrassenfläche gelegenen Kiesgrube Holzkohlen aus einer Lehmscholle in ca. $3 \mathrm{~m}$ Tiefe unter Geländeoberfläche geborgen werden. Das ${ }^{14} \mathrm{C}$-AMS-Alter von $5900 \pm 40$ a BP weist ebenfalls auf eine Ausbildung der Mundrachinger Stufe während des Atlantikums hin. Im Einklang damit steht das ${ }^{14} \mathrm{C}$-AMS-Alter einer Schneckenschale mit einem Alter von $5720 \pm 40$ a BP aus einer in $3 \mathrm{~m}$ Tiefe unter Geländeoberfläche eingelagerten Lehmscholle in der Kiesgrube „Kolonie Obermeitingen“ etwa $1 \mathrm{~km}$ weiter talaufwärts der oben beschriebenen Lokalitäten.

\subsection{Die Obere und Untere Lorenzbergstufe}

Die Obere und die Untere Lorenzbergstufe wurden erstmalig von BRUNNACKer (1964) beschrieben und von Diez (1968) übernommen. Am locus typicus, dem Lorenzberg, sind sie allerdings lediglich als zwei kleine Terrassenreste erhalten. So steht die Kapelle des Lorenzbergs auf der Oberen Lorenzbergstufe, während der etwa $3 \mathrm{~m}$ niedrigere nordöstliche Bereich des Lorenzberges zur Unteren Lorenzbergstufe gehört. Nach Brunnacker (1964) besitzen beide Terrassen eine unterschiedliche Tiefenlage ihrer Terrassenbasis über Molasseablagerungen. Im Untersuchungsgebiet sind beide Stufen aufgrund der Nähe zu den jungholozänen Lechauen nur in kleinen Terrassenresten erhalten (Abb. 2a, Abb. 2b).

Die quartären Schottermächtigkeiten erreichen hier nur 2 bis $6 \mathrm{~m}$, so dass davon auszugehen ist, dass die Quartärbasis der Terrassenbasis entspricht. Informationen über das Schichtungsbild des Terrassenkörpers der Lorenzbergstufen liegen nicht vor. Beide Lorenzbergstufen können kleinräumig von bis zu $1 \mathrm{~m}$ mächtigen Flussmergeln bedeckt sein, auf denen unter Wald nach Diez (1968) Braunerden mit geringer Entwicklungstiefe verbreitet sind. Absolute Altersdaten liegen aus beiden Lorenzbergstufen bisher nicht vor. Aufgrund ihrer Lage im Tal sind sie älter als die Seestallstufen und die römerzeitliche Ältere Auenstufe und jünger als die im Atlantikum entstandene Stufe von Mundraching.

\subsection{Die Obere und Untere Seestallstufe}

Die Obere und die Untere Seestallstufe konnten erstmals im Rahmen dieser Kartierung nachgewiesen werden. Bei DiEz (1973) erfuhren beide Terrassenniveaus meist eine Einstufung als Stufe von Pitzling, bereichsweise auch als Stufe von Spötting. Der locus typicus ist der Ort Seestall, der sich über beide Stufen hinweg erstreckt. Höhenmäßig liegen beide Seestallstufe zwischen den Lorenzbergstufen und den Auenstufen. Dabei ist die Oberfläche der Oberen Seestallstufe etwa 1,5 m höher als die der Unteren Seestallstufe. Beide Terrassen sind im Untersuchungsgebiet flächenmäßig am wenigsten vertreten (Abb. 2a). Ein ausgedehnteres und durch mehrere niedrige Geländestufen gegliedertes Terrassenareal der Unteren Seestallstufe erstreckt sich zwischen Seestall und Dornstetten, südlich Landsberg.

Aussagen über das Oberflächengefälle beider Terrassenstufen können wegen ihrer kleinräumigen Erhaltung

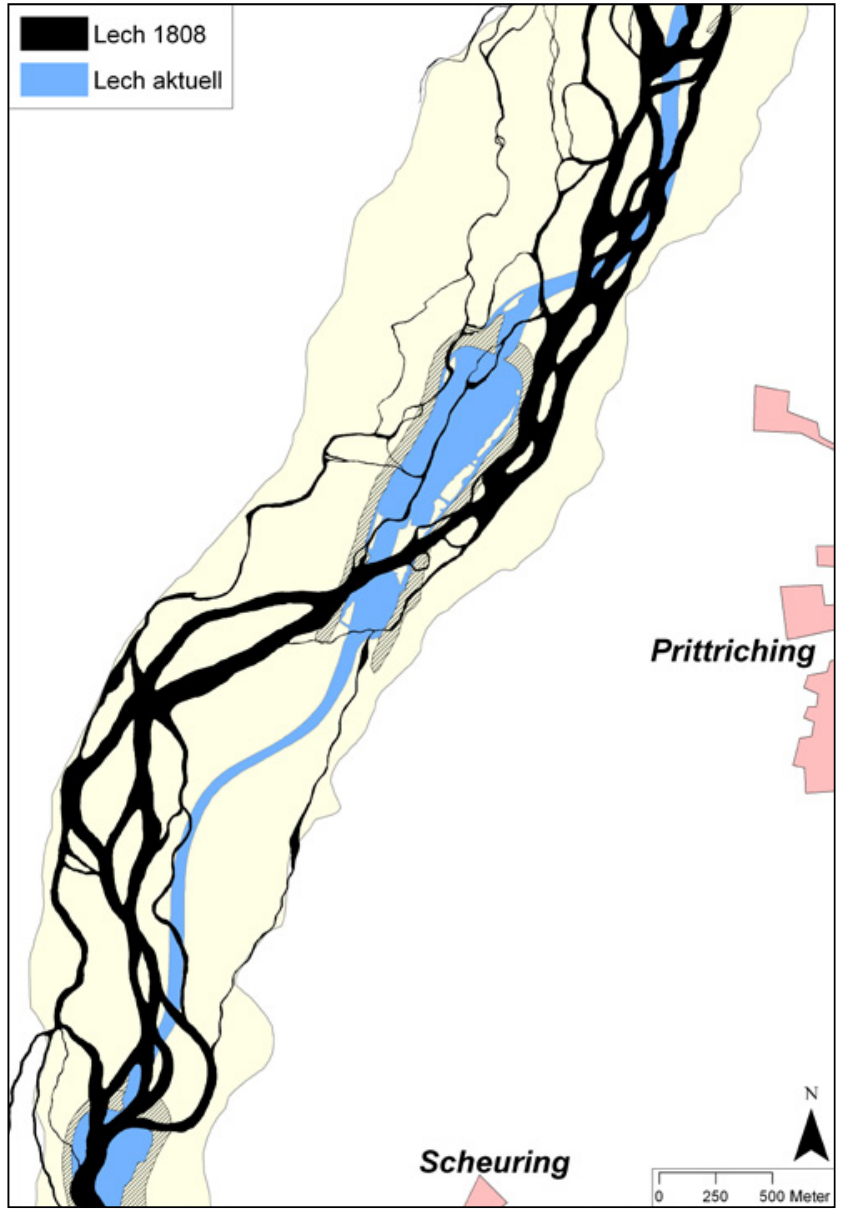

Abb. 5: Lechlauf um 1808, vor den Flusskorrekturen des 19. und 20. Fahrhunderts auf Basis der Urkatasteraufnahmen Bayerns.

Fig. 5: Lech at about 1808 before river regulations in the 19th and 20th century based on "Urkatasteraufnahmen Bayerns".

lediglich für den Talraum zwischen Seestall und Dornstetten getroffen werden. Dort beträgt es etwa $3 \%$ und liegt damit in einer Größenordnung, die den anderen holozänen Terrassen entspricht. Die Quartärbasis wird im Mittel bereits bei 2 bis $7 \mathrm{~m}$ unter Geländeoberfläche erreicht, so dass davon auszugehen ist, dass diese auch der Terrassenbasis entspricht. Wegen fehlender Aufschlüsse gibt es keine Informationen über den lithologischen Aufbau des Terrassenkörpers. Die Obere Seestallstufe besitzt in der Regel keine sandige Auenmergeldecke, während auf der Unteren Seestallstufe häufiger Hochflutauflagen aus bis zu $30 \mathrm{~cm}$ mächtigen Mergeln, Sanden und Feinkiesen verbreitet sind. Absolute Altersdaten liegen aus beiden Seestallstufen bisher nicht vor. Aufgrund ihrer Lage im Tal sind sie älter als die römerzeitliche Auenstufe und jünger als die beiden Lorenzbergstufen.

\section{5 Älter, Jüngere und Jüngste Auenstufe}

Beiderseits des Lechs sind bis zu drei jungholozäne Auenstufen erhalten. Wegen der ähnlichen Höhenlage ihrer Oberflächen ist eine Zuordnung isolierter Terrassenreste schwierig, so dass für deren Einstufung jeweils das morphostratigraphische Mindestalter gewählt wurde. Vor allem südlich von Landsberg sind die Auenstufen nur noch fragmentarisch erhalten, da große Areale durch den Bau 
der Staustufen überflutet wurden. Größere Auengebiete sind dort bei Epfach, Mundraching und Dornstetten sowie flussabwärts bei Pitzling und Landsberg erhalten (Abb. 2a). Unterhalb der Staustufe Kaufering bilden diese Auenterrassen dann einen bis an den Nordrand des Untersuchungsgebietes reichenden, $15 \mathrm{~km}$ langen und den Lech in durchschnittlich $800 \mathrm{~m}$ Breite beiderseits begleitenden Streifen (Abb. 2b).

Im südlichen Untersuchungsgebiet bei Epfach bilden die drei Auenstufen eine morphologische Terrassentreppe mit einem Höhenunterschied von insgesamt $3 \mathrm{~m}$ zwischen der am höchsten gelegenen römerzeitlichen Terrasse und der am niedrigsten gelegenen neuzeitlichen Terrasse. Weiter talabwärts nähern sich deren Oberflächenniveaus zunehmend aneinander an (Abb. 3). Unterhalb von Kaufering sind sie fast höhengleich, tragen zudem in großen Arealen Auwald und können daher fast nur über den Verlauf ihrer primären Aurinnenscharen morphologisch abgegrenzt werden. Dabei zeigt insbesondere die jüngste Auenstufe eine starke Reliefierung von sich verzahnenden Aurinnensystemen, Paläoflussbetten, Strudellöchern und ausgeprägten Sand- und Kiesrücken. In ihrem Relief spiegelt sich das junge Alter einer teilweise noch im 19. und 20. Jahrhundert aktiv gestalteten und von zahlreichen Einzelarmen durchzogenen Flusslandschaft wieder (Abb. 5). Typisch für diese im aktuellen Hochflutbereich liegenden Terrassenoberflächen sind Auenmergeldecken mit Mächtigkeiten von mehreren Dezimetern. In ehemaligen Flussrinnen treten mächtigere Aurinnenfüllungen in Größenordnungen von bis zu $2 \mathrm{~m}$ auf. Die Kieskörper der Auenstufen, die teilweise bis an die Terrassenoberfläche reichen, erreichen Mächtigkeiten von bis zu $10 \mathrm{~m}$. Über deren Aufbau und Schichtungsbild liegen mangels Aufschlüssen keine Informationen vor.

Archäologische Funde am Fuße des Lorenzbergs bei Epfach weisen nach DiEz (1968) der „Älteren Auenstufe“ eine Bildung in der römischen Kaiserzeit zu. Im Bereich der Altstadt von Landsberg findet man auf der Älteren Auenstufe bereits hochmittelalterliche Gebäudereste. Die jüngste Auenstufe konnte mit Hilfe historischer Flurkarten aus der ersten Hälfte des 19. Jahrhunderts altersmäßig in die späte Neuzeit eingestuft werden. Für die jüngere Auenstufe wird eine Entstehung im Mittelalter angenommen.

\section{Schlussfolgerungen}

Insgesamt konnte durch die Neuaufnahmen gezeigt werden, dass die Terrassenlandschaft des Lechs aus mindestens 17 jungquartären Terrassenstufen mit drei bisher nicht bekannten Terrassen, der Stufe von Mundraching und den Stufen von Seestall, besteht. Sämtliche Stufen bilden in der Regel eine Terrassentreppe, deren einzelne Terrassen durch markante Geländestufen voneinander abgesetzt sind. Infolge nachträglicher Erosion sind nirgendwo im Untersuchungsgebiet alle Terrassenstufen in unmittelbarer kontinuierlicher Abfolge erhalten. Die älteste, vermutlich würmzeitliche, Lechterrasse bildet dabei die schmale, lössbedeckte Terrassenleiste der Übergangsterrasse südlich von Landsberg. Aufgrund ihrer Höhenlage und Lössbedeckung kann von einem mindestens mittelwürmzeitlichen Alter ausgegangen werden. Die ältesten Lechterrassen, die letztlich im Talgrund dominieren, bestehen im Arbeits- gebiet aus mindestens drei hoch- und späthochglazialen Niederterrassen (HNT, Stufe von Altenstadt, Stufe von Schongau-Peiting) der Würm-Kaltzeit. Die nächstjüngeren Lechterrassen sind die Stufe von Unterigling, die Zwischenstufe und die Stufe von Friedheim. Bei der Stufe von Unterigling konnte auf Basis zweier ${ }^{14} \mathrm{C}$-Datierungen eine spätglaziale Alterstellung belegt werden. Das widerspricht den Vermutungen bisheriger Bearbeiter (Troll 1925, DiEz 1968), die die Stufe von Unterigling ins späte Würmhochglazial einstuften. Sowohl die Zwischenstufe als auch die Stufe von Friedheim wurden bereits von Diez (1968) als spätglaziale Bildungen angesehen. Die ${ }^{14} \mathrm{C}$-Datierung einer Schneckenschale aus einer eingelagerten Lehmscholle deutet für die Stufe von Friedheim auf eine Bildungszeit am Ausgang der Jüngeren Dryaszeit hin. Unklar ist, ob die Obere Epfachstufe ebenfalls noch eine spätglaziale oder schon eine präboreale Lechterrasse ist. Die anschließend gebildeten, treppenartig angeordneten neun holozänen Lechterrassen entstanden im Zeitraum vom Präboreal bis zur weitgehenden Flusslaufregulierung im 19. Jahrhundert. Erste ${ }^{14} \mathrm{C}$-Datierungen deuten auf ein präboreales Alter der Unteren Epfachstufe und ein atlantisches Alter der Stufe von Mundraching hin. Archäologische Befunde bei Epfach belegen für die Ältere Auenstufe ein römerzeitliches Alter, historische Flurkarten für die Jüngste Auenstufe eine Bildung seit dem frühen 19. Jahrhundert.

\section{Danksagung}

Die dargestellten Kartierungen wurden im Rahmen des geologischen Projektes „Informationsoffensive Oberflächennahe Geothermie 2008-2011“ im Auftrag des Landesamtes für Umwelt (LfU) in Bayern durchgeführt. Hier möchten sich die Autoren für die umfassende finanzielle und logistische Unterstützung durch das LfU Bayern bedanken. Ein besonderer Dank geht dabei an Dr. G. Doppler und Dr. E. Kroemer für deren großes Engagement und Diskussionsbereitschaft. Für die Bereitstellung von Schichtenverzeichnissen von Bohrungen sei den beteiligten Wasserwirtschafts- und Straßenbauämtern gedankt. Wir danken Dr. W. Grottenthaler für seine engagierte Durchsicht der vorliegenden Arbeit und seine hilfreichen Ergänzungen.

\section{Literatur}

BAuer, F. (1979): Das flußmorphologische Verhalten des bayerischen Lechs. - Schriftenreihe des Bayerischen Landesamtes für Wasserwirtschaft, 9; München.

Brunnacker, K. (1959): Zur Kenntnis des Spät- und Postglazials in Bayern. - Geologica Bavarica, 43: 74-150; München.

BrunNACKer, K. (1964): Die geologisch-bodenkundlichen Verhältnisse bei Epfach. - Münchner Beiträge zur Vor- und Frühgeschichte, 7: 140-156; München.

DiEz, T. (1968): Die würm- und postwürmglazialen Terrassen des Lech und ihre Bodenbildungen. - Eiszeitalter und Gegenwart, 19: 102-128; Öhringen.

Diez, T. (1973): Geologische Karte von Bayern 1:25.000, Erläuterungen zum Blatt Nr. 7931 Landsberg am Lech. - 78 S.; München (Bayerisches Landesamt für Umwelt).

Gesslein, B. \& Schellmann, G. (2010): Zur Stratigraphie und Altersstellung der jungquartären Lechterrassen zwischen Kinsau und Prittriching - erste Ergebnisse. - Bamberger Geographische Schriften, 24: 189-213; Bamberg.

Grottenthaler, W. (1993): Geologische Karte von Bayern 1:25 000, Blatt Nr. 8131 Schongau. - München (Bayerisches Geologisches Landesamt). 
Grottenthaler, W. (2009): Geologische Karte von Bayern 1:25 000, Er läuterungen zum Blatt Nr. 8131 Schongau. - München (Bayerisches Landesamt für Umwelt).

KNAUER, J. (1929): Erläuterungen zum Blatt München-West (Nr. XXVII) der Geognostischen Karte von Bayern 1:100 000, Teilblatt Landsberg. - 47 S.; München.

RathJENs, C. (1951): Über die Zweiteilung der Würmeiszeit im nördlichen Alpenvorlande. - Petermanns Geographische Mitteilungen, 95 89-97; Gotha.

Schirmer, W. (1983): Die Talentwicklung an Main und Regnitz seit dem Hochwürm. - In: Geologisches Jahrbuch. Holozäne Talentwicklung Methoden und Ergebnisse, 71: 11-43; Hannover.
Schreiber, U. (1985): Das Lechtal zwischen Schongau und Rain im Hoch-, Spät- und Postglazial. - Geologisches Institut der Universität Köln Sonderveröffentlichung, 58: 192 S.; Köln.

Troll, K. (1925): Die Rückzugsstadien der Würm-Eiszeit im nördlichen Vorland der Alpen. - Mitteilungen der Geographischen Gesellschaft München, 18: 281-292; München.

Troll, K. (1926): Die jungglazialen Schotterfluren im Umkreis der deutschen Alpen. - Forschungen zur deutschen Landes- und Volkskunde, 24 (4): 158-256; Stuttgart.

WiLKE, B. M. (1975): Bodenchronosequenzen aus Lockersedimenten der Lechterrassen. - Zeitschrift für Pflanzenernährung und Bodenkunde, 2: 153-171; Weinheim 